\title{
Connecting black holes and galaxies in faint radio populations at cosmic noon
}

\author{
Stacey Alberts ${ }^{1}\left(\mathbb{D}\right.$, Wiphu Rujopakarn $^{2}$ and George H. Rieke ${ }^{1}(\mathbb{D}$ \\ ${ }^{1}$ Steward Observatory, University of Arizona, 933 North Cherry Avenue, \\ Tucson, AZ 85721, USA \\ email: salberts@email.arizona.edu \\ ${ }^{2}$ Department of Physics, Faculty of Science, Chulalongkorn University \\ 254 Phayathai Road, Pathumwam, Bangkok 10330, Thailand
}

\begin{abstract}
We leverage new ultra-deep, high resolution, multi-frequency radio imaging at 6 and $3 \mathrm{GHz}$ with the unique datasets available in the GOODS-S/HUDF region in order to assess the AGN fraction in a faint radio-selected sample. For AGN identification, we adopt a multi-wavelength approach, combining X-ray and (mid-)infrared (IR) selections with radio identification such as X-ray to radio excess, flat radio spectral slopes, and the radio-IR correlation. We identify AGN in $43 \%$ of our radio sample, yielding an AGN source density of $\sim 1 \mathrm{arcmin}^{-2}$. This AGN fraction is likely underestimated, as 1) our shallower $3 \mathrm{GHz}$ data is biased against flat radio spectrum sources and 2) all of our selections may be biased against the most heavily obscured AGN. The James Webb Space Telescope's Mid-Infrared Instrument (MIRI) will address the latter issue and we briefly outline our Cycle 1 Guaranteed Time Observation (GTO) program to search for heavily obscured AGN.
\end{abstract}

Keywords. Galaxy: evolution, radio continuum: galaxies, galaxies: active

\section{Introduction}

Understanding the role of supermassive black hole growth in shaping galaxy populations across cosmic time remains an open and pressing issue. Locally, the tight relation between black hole mass and galaxy properties has long been established (e.g., Magorrian et al. 1998), a link which is supported at earlier epochs by the strikingly similar evolution of the volume-averaged cosmic star formation (SF) and black hole accretion histories (see Madau \& Dickinson 2014, for a review). Though existing on very disparate physical scales, SF and Active Galactic Nuclei (AGN) activity may be connected through a common gas supply (Vito et al. 2014), with AGN playing a key role in the self-regulation of massive galaxy growth through feedback (e.g. Kormendy \& Ho 2013). However, establishing the causal link between these phenomena remains challenging, in no small part hampered by difficulties in obtaining a complete census of AGN within a given statistical galaxy population. AGN selection methods are well known to be sensitive to distinct AGN subpopulations, with minimal overlap (Mendez et al. 2013; Delvecchio et al. 2017). This necessitates a multi-wavelength approach to avoid strong selection biases.

In this study, we leverage new multi-frequency, ultra-deep, high resolution radio imaging with the deep X-ray-mid-infrared (IR) imaging and spectroscopy available in the GOODS-S/HUDF region in order to obtain a census of AGN within faint radio galaxies and place constraints on the AGN population that may still be missing due to extreme obscuration (see Hickox \& Alexander 2018, for a review). Radio imaging provides a unique complement to common X-ray, optical, and (mid-)IR AGN selections, supplying an extinction-free tracer of SF and/or AGN activity, with multiple AGN indicators: 


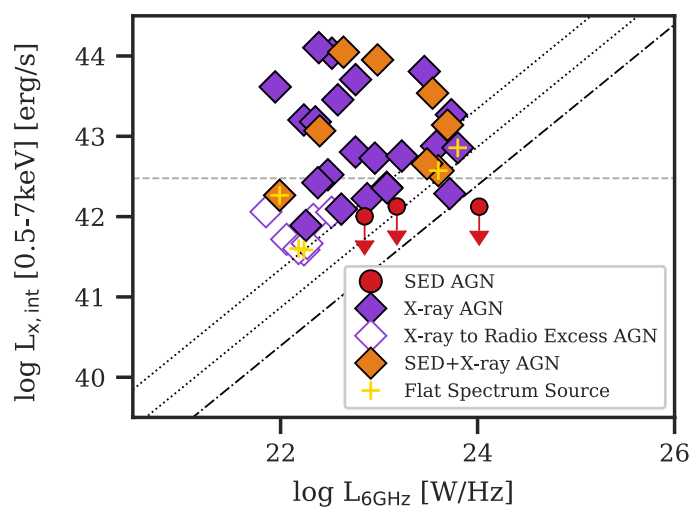

Figure 1. The intrinsic (absorption corrected, see Luo et al. 2017) 0.5-7 keV X-ray luminosity as a function of $6 \mathrm{GHz}$ luminosity for radio sources classified as AGN. Multiple selection techniques are used: X-ray properties (diamonds), optical-mid-IR SED decomposition (orange diamonds and red circles), and flat radio spectrum sources (yellow crosses). The threshold for AGN selection via high intrinsic X-ray luminosity (dashed) as well as the expected $L_{x}-L_{6 \mathrm{GHz}}$ relation for SFGs (dot-dashed) and 3 and $6 \sigma$ outliers (dotted) from this relation are indicated.

radio jets, flat (radio) spectrum sources, and outliers from the radio-IR correlation (see Padovani 2016, for a review).

\section{Data}

Our sample is selected at $6 \mathrm{GHz}$ using deep C-band (4-8 GHz) imaging from the Karl G. Jansky Very Large Array within the half-power radius of a single pointing centered on the Hubble Ultra Deep Field (Rujopakarn, in prep.). This imaging reaches a depth of $0.32 \mu \mathrm{Jy}$ beam $^{-1}$ with an angular resolution of $0.61^{\prime \prime} \mathrm{x} 0.31^{\prime \prime}, \sim 10$ times deeper and $\sim 2$ times higher resolution then comparable surveys (i.e. Delvecchio et al. (2017); Gim et al. (2019)). The data reduction and source extraction will be presented in Rujopakarn, in prep. Our $6 \mathrm{GHz}$ sample is then matched to the Chandra X-ray Observatory $7 \mathrm{Ms}$ GOODS-S catalog (Luo et al. 2017) and the UV-mid-IR 3D-HST (v4.1) photometric catalog (Skelton et al. 2014), providing X-ray properties, $0.3-24 \mu \mathrm{m}$ photometry, redshifts, and stellar masses. Our final sample is $1006 \mathrm{GHz}$ sources with secure counterparts at $z \sim 0.75-3$. Lastly, this sample is matched to new $3 \mathrm{GHz} \mathrm{S}$-band (2-4 GHz) imaging (Rujopakarn, in prep.), centered on the same area, which reaches a depth of $0.7 \mu \mathrm{Jy}_{\text {beam }}{ }^{-1}$ and an angular resolution of $0.7^{\prime \prime} .75 / 100$ of the $6 \mathrm{GHz}$ sources have a robust $3 \mathrm{GHz}$ counterpart.

\section{AGN Selection}

$X$-ray Selection: X-ray based AGN classifications are adopted following the criteria outlined in Luo et al. (2017). Briefly, these are 1) a high intrinsic X-ray luminosity, 2) a hard X-ray spectrum indicating obscured AGN, 3) excess X-ray to optical or near-IR emission, and 4) excess X-ray to radio emission.

Out of our 100 6GHz sources, 31 are classified as AGN in Luo et al. (2017). Their intrinsic X-ray luminosity as a function of radio luminosity (assuming $\alpha=-0.7$; Condon 1992) can be seen in Figure 1. While most of the X-ray selected AGN satisfy the high intrinsic $\mathrm{X}$-ray luminosity criteria, a threshold chosen to lie comfortably above the potential X-ray emission generated by SF, about a quarter are identified via X-ray excess relative to optical, near-IR, or radio emission. We expand on the latter criteria - X-ray to radio excess 
- with our $6 \mathrm{GHz}$ imaging, a factor of 9 deeper than the radio used in Luo et al. (2017). To do so, we look for sources with a significant excess $(>5 \mathrm{x})$ of X-ray emission over the expected level from pure SF given the $L_{\mathrm{x}}-L_{\text {radio }}$ relation for star forming galaxies (SFGs) (i.e. Alexander et al. 2005; Lehmer et al. 2016). We find an additional 7 AGN candidates based on this criteria.

Optical-mid-IR Selection via SED Fitting: Mid-IR color-color diagnostics (i.e. Stern et al. 2005) have proven a useful tool in identifying AGN sub-populations which are often missed in X-ray surveys due to obscuration. These color diagnostics isolate regions of a galaxy spectrum where obscured AGN may dominate over the host galaxy emission. Optical-mid-IR SED fitting is an expansion on this technique, utilizing photometric data over a long baseline to identify AGN (i.e. Assef et al. 2010; Chung et al. 2014; Delvecchio et al. 2017.

In this work, we utilize the extensive optical-mid-IR coverage provided by 3D-HST (Skelton et al. 2014) and perform SED fitting and decomposition through an adapted version of the publicly available code from Assef et al. (2010). SED fits are performed as a non-negative linear combination of templates over the range $0.3-30 \mu \mathrm{m}$, fitting galaxy templates first and then galaxy+AGN templates. An F-test is then applied to see if the AGN template significantly improves the fit (see Chung et al. (2014) for details). We identify 12 AGN via this method, 3 of which where not previously identified (orange diamond and red circles, Figure 1). Our X-ray identified AGN fraction and overlap between X-ray and optical-mid-IR selection is high relative to other studies (i.e. Delvecchio et al. 2017) due to the uniquely deep and complete X-ray dataset available in the GOODS-S/HUDF region.

Radio Selection via Jets: There are no large scale radio jets apparent in our radio imaging as determined via visual inspection.

Radio Selection via the Radio Spectral Index: The low frequency radio spectrum of SFGs is typically parameterized as a superposition of two power laws: the dominant steep synchrotron spectrum and a flatter free-free component contributing $\sim 10 \%$ at $1.4 \mathrm{GHz}$ (Condon 1992). For AGN with compact, optically thick radio cores, the radio spectrum in SFGs a synchrotron spectrum, $S_{\nu} \propto \nu^{\alpha}$ with $\alpha \sim-0.7$ arising from the acceleration of cosmic rays in supernova remnants - experiences synchrotron self-absorption, producing a telltale flat spectrum with $\alpha \geq-0.5$ (Padovani 2016).

In Figure 2, the 3-6 GHz radio spectral slope, $\alpha_{6 \mathrm{GHz}}^{3 \mathrm{GHz}}$, is shown for our AGN. We identify 8 flat spectrum sources, including 2 which were not selected as AGN via other methods, constituting $18 \%$ of our AGN. This number is likely a lower limit on the fraction of flat spectrum sources as one quarter of our radio sample are not detected at $3 \mathrm{GHz}$. Additional $3 \mathrm{GHz}$ imaging is being gathered by our team; in the meantime, we stack the $3 \mathrm{GHz}$ undetected sources (Figure 2, green star), finding that their average slope is $\alpha_{6 \mathrm{GHz}}^{3 \mathrm{GHz}} \sim 0$, suggesting that there are indeed additional flat spectrum sources in our sample.

Radio Selection via Radio-Infrared Correlation: The radio-IR correlation (i.e. Yun et al. 2001) is a tight relation for SFGs arising due to the similar origins of radio and IR emission, with radio synchrotron generated via supernova remnants and IR from dustreprocessed starlight from young stars. In the presence of an AGN, the radio may be elevated relative to the IR and such sources identified as outliers from the radio-IR correlation. Here we compare radio emission with the observed $24 \mu \mathrm{m}$ flux via the parameter $\mathrm{q}_{24, \mathrm{obs}}=\log \left(S_{24 \mu \mathrm{m}, \mathrm{obs}} / S_{\text {radio }}\right)$ (i.e. Bonzini et al. 2013). In order to define $\mathrm{q}_{24, \mathrm{obs}}$ for SFGs, we adopt the Rieke et al. (2009) empirical local templates with $\log \mathrm{L}_{\mathrm{IR}}=10-13 \mathrm{~L}_{\odot}$ with 0.2 dex scatter. Figure 3 shows $\mathrm{q}_{24, \text { obs }}$ for our radio sample up to $z \sim 3$, beyond 


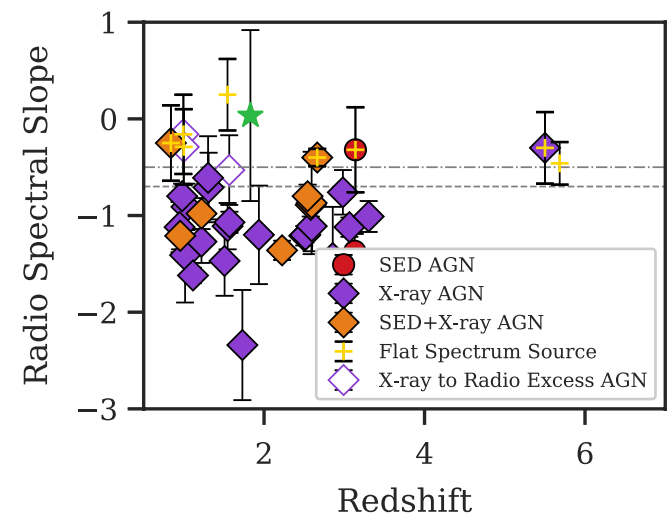

Figure 2. The radio spectral slope as a function of redshift for AGN. Symbols and colors are as in Figure 1. The green star is the stacked radio spectral slope of sources undetected at $3 \mathrm{GHz}$. The dashed line indicates the slope typically assumed for SFGs $(\alpha=-0.7$; Condon 1992), while the dot-dashed line indicates the threshold for flat spectrum AGN $(\alpha \geqslant-0.5$; Padovani 2016).

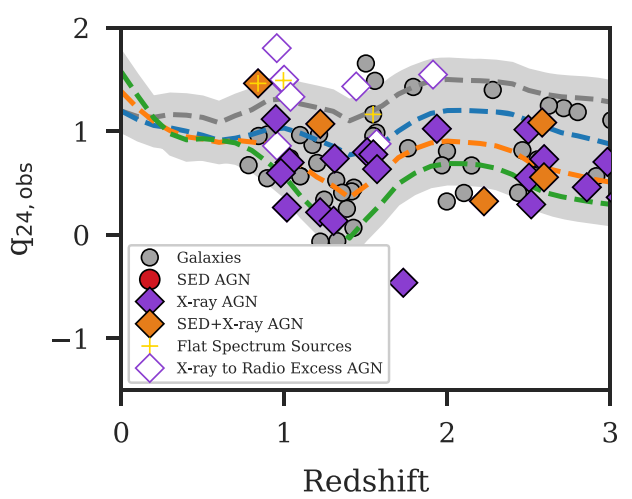

Figure 3. The radio-IR correlation, parameterized via $\mathrm{q}_{24 \mu \mathrm{m}, \mathrm{obs}}$ as a function of redshift for our radio sample, including SFGs (gray circles). Symbols and colors are as in Figure 1. The dashed lines represent $\mathrm{q}_{24 \mu \mathrm{m} \text {,obs }}$ for the Rieke et al. (2009) local templates with $\log \mathrm{L}_{\mathrm{IR}}=10$, $11,12,13 \mathrm{~L}_{\odot}$ with 0.2 dex scatter (shaded regions).

which $24 \mu \mathrm{m}$ no longer traces SF. We find only one source, previously identified as an $\mathrm{X}$-ray AGN, is an outlier from the SFG distribution.

\section{A Census of AGN}

We identify AGN in 43/100 sources in our radio sample, an AGN source density of $~ 1$ $\operatorname{arcmin}^{-2}$. This fraction is a lower limit pending our approved program for deeper $3 \mathrm{GHz}$ data, which may reveal additional flat spectrum sources.

What AGN might we be missing? The contribution of heavily obscured and Compton Thick (CT) AGN to the total AGN population is still disputed, with current estimates at $\sim 30-50 \%$ (Hickox \& Alexander 2018). Exploiting our deep X-ray imaging and SED fitting, we compare our sources to the intrinsic relation (Lansbury et al. 2015) between X-ray luminosity and the $6 \mu \mathrm{m}$ AGN luminosity - measured via SED decomposition - in Figure 4. We find that by this measure approximately a quarter of our AGN are heavily obscured or CT, slightly below the lower end of estimates of the CT fraction. For a complete analysis of these results, the reader is referred to Alberts et al., in prep. 


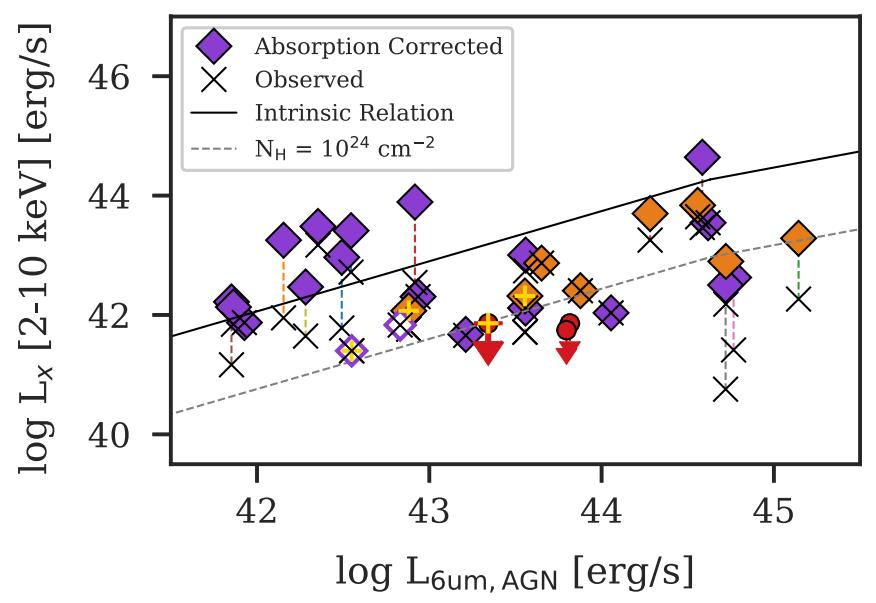

Figure 4. The X-ray luminosity (converted to $2-10 \mathrm{keV}$ ) as a function of the luminosity at $6 \mu \mathrm{m}$, derived from SED decomposition for our AGN. Symbols represent the absorption corrected X-ray (Luo et al. 2017), as in Figure 1. X's mark the observed X-ray luminosity. The intrinsic $L_{\mathrm{x}}-L_{6 \mu \mathrm{m}}$ relation for unobscured AGN (solid) and the relation given heavy obscuration $\left(\mathrm{N}_{\mathrm{H}}=10^{24} \mathrm{~cm}^{-2}\right)$ (dashed; i.e. Lansbury et al. 2015) is indicated.

\section{Finding Obscured AGN with Future Facilities}

A complete accounting of the AGN population and their influence on their host galaxies remains elusive, in part because of the difficulty in identifying obscured and Compton Thick AGN in large, unbiased photometric samples. A solution to this problem will be available upon the launch of the James Webb Space Telescope (JWST). JWST's MidInfrared Instrument (MIRI) will provide 9 photometric bands covering $5.6-25.5 \mu \mathrm{m}$ with high sensitivity and resolution (Bouchet et al. 2015). This wavelength coverage is ideally suited to identify AGN through mid-IR diagnostics (i.e. Kirkpatrick et al. 2017). This capability is the focus of our Cycle 1 GTO HUDF imaging program (PI: G. Rieke), which samples the full MIRI photometric coverage in order to take advantage of the ubiquitous minimum in stellar emission found in non-AGN galaxies at rest 3-5 $\mathrm{m}$ (Sorba \& Sawicki 2010). In the presence of an AGN, even heavily obscured, this minimum will be filled in by warm torus emission, providing a new and highly effective AGN selection technique that will enable a more complete census of AGN up to cosmic noon.

\section{Acknowledgements}

The authors thank Kristina Nyland and Preshanth Jagannathan for their work on the radio imaging described in this proceedings.

\section{References}

Alexander, D. M., Bauer, F. E., Chapman, S. C., et al. 2005, ApJ, 632, 736

Assef, R. J., Kochanek, C. S., Brodwin, M., et al. 2010, ApJ, 713, 970

Bonzini, M., Padovani, P., Mainieri, V., et al. 2013, MNRAS, 436, 3759

Bouchet, P., García-Marín, M., Lagage, P.-O., et al. 2015, PASP, 127, 612

Condon, J. J. 1992, ARA\&A, 30, 575

Chung, S. M., Kochanek, C. S., Assef, R., et al. 2014, ApJ, 790, 54

Delvecchio, I., Smolčić, V., Zamorani, G., et al. 2017, A\&SA, 602, A3

Elvis, M., Wilkes, B. J., McDowell, J. C., et al. 1994, ApJS, 95, 1

Gim, H. B., Yun, M. S., Owen, F. N., et al. 2019, ApJ, 875, 80

Hickox, R. C. \& Alexander, D. M. 2018, ARA $\& A$ A, 56, 625

Kirkpatrick, A., Alberts, S., Pope, A., et al. 2017, ApJ, 849, 111 
Kormendy, J. \& Ho, L. C. 2013, ARA\&A, 51, 511

Lansbury, G. B., Gandhi, P., Alexander, D. M., et al. 2015, ApJ, 809, 115

Lehmer, B. D., Basu-Zych, A. R., Mineo, S., et al. 2016, ApJ, 825, 7

Luo, B., Brandt, W. N., Xue, Y. Q., et al. 2017, ApJS, 228, 2

Madau, P. \& Dickinson, M. 2014, ARA\&A, 52, 415

Magorrian, J., Tremaine, S., Richstone, D., et al. 1998, AJ, 115, 2285

Mendez, A. J., Coil, A. L., Aird, J., et al. 2013, ApJ, 770, 40

Padovani, P. 2016, A\&SA Rev., 24, 13

Rieke, G. H., Alonso-Herrero, A., Weiner, B. J., et al. 2009, ApJ, 692, 556

Skelton, R. E., Whitaker, K. E., Momcheva, I. G., et al. 2014, ApJS, 214, 24

Sorba, R. \& Sawicki, M. 2010, ApJ, 721, 1056

Stern, D., Eisenhardt, P., Gorjian, V., et al. 2005, ApJ, 631, 163

Tisanić, K., Smolčić, V., Delhaize, J., et al. 2019, A\&\&A, 621, A139

Vito, F., Maiolino, R., Santini, P., et al. 2014, MNRAS, 441, 1059

Yun, M. S., Reddy, N. A., \& Condon, J. J. 2001, ApJ, 554, 803 